\title{
Special issue on consolidation, reconsolidation and extinction
}

\author{
H. Valerie Curran • Trevor W. Robbins
}

Published online: 14 March 2013

(C) Springer-Verlag Berlin Heidelberg 2013

The notion that our memories are not fixed but evolve and change over time can be traced back to Ribot (1881). He noted in his classic monograph (Les Maladies de la Mémoire) how amnesic patients often had little memory of their recent past but could remember many things from way back in their lives. Ribot suggested that a biological process unfolds over time which makes memories progressively stronger so that traumatic brain injury disrupts recollection of the recent but not distant past. Subsequently, Muller and Pilzecker (1900) put forward a cognitive 'retroactive interference' account of the same phenomenon whereby shortly after new information is encoded, it could be disrupted by learning other similar information. It is now accepted that memories undergo multiple processes of consolidation. Immediately after learning, memories are labile and can be disrupted by interference, amnestic drugs or trauma. In the hours after learning, they are 'consolidated' at the neuronal level and over time become increasingly stabile and cortically distributed through a process of 'systems consolidation'.

Memory consolidation involves a brief cascade of molecular, cellular and epigenetic events that alter synaptic efficiency followed by more prolonged systems-level interaction between the hippocampus (which stores new memories) and areas of the cerebral cortex (supporting older memories; e.g. McGaugh 2000). There is now considerable evidence that for a short while after learning — during the consolidation interval - memories are vulnerable to disruption not only by new learning of other material but also by protein synthesis inhibitors like anisomycin,

H. V. Curran $(\bowtie)$

Clinical Psychopharmacology Unit, University College London,

Gower Street,

London WC1E 6BT, UK

e-mail: v.curran@ucl.ac.uk

T. W. Robbins

Department of Psychology, and Behavioural

and Clinical Neuroscience Institute,

University of Cambridge, Downing Street,

Cambridge CB2 3EB, UK

e-mail: twr2@cam.ac.uk electroconvulsive shock, beta-blockers and $N$-methyl D-aspartate (NMDA) antagonists. Disruption does not occur outside this consolidation interval, suggesting that these memories are in a fixed, consolidated, stable state and remain so potentially indefinitely (McGaugh 1966; Squire and Alvarez 1995).

Over the past decade, there has been an accumulation of evidence that previously consolidated memories can, in certain circumstances, also be disrupted or even enhanced (c.f. Nader et al. 2000; Dudai 2006; Hardt et al. 2010; Milton and Everitt 2012). In such conditions, those established memories can be made labile again through the reactivation of the memory trace. This reactivation - brought about by associated environmental cues or re-presentation of learned material - and associated memory retrieval can initiate a period of instability during which the memory itself can be strengthened or updated or disrupted prior to being reconsolidated. Similarly, reconsolidation can be chemically disrupted, resulting in potential memory erasure. Just as protein synthesis is required for consolidation of memories, it is also essential for the reconsolidation of memories after reactivation. As Nader et al.'s (2000) landmark study showed, blocking protein synthesis by infusing anisomycin into the basolateral amygdala of rats after reactivating a cued fear memory drastically reduced fear behaviour (freezing) to the cue the next day and for 14 days after.

Retrieval can thus return memories to a plastic, malleable state. What role reconsolidation plays in real life is still debated (Dudai 2006). From an evolutionary perspective, reconsolidation could serve an important adaptive role of updating prior knowledge at the time of retrieval to maintain the predictive validity of memories. For example, updating of memory to incorporate changes in environmental dangers can be critical to an animal's survival. Indeed, prediction error-based mismatch between expected and actual outcomes appears to be a necessary condition for memory reconsolidation (Osan et al. 2011; Sevenster et al. 2013). As human cognitive function uses memory to interpret and act upon current events and envisage future events, memory updating is an essential means of keeping memory relevant. The other side of this coin is that, by 
returning a memory to a plastic state, reactivation can also allow for misleading new information to be incorporated into old memory and produce memory distortions seen for example in Loftus' (1979) classic studies of eyewitness testimony. In extreme cases, a memory can be constructed of an event that was never experienced.

At the same time, returning a memory to a labile state might be beneficial in providing an opportunity to rewrite aberrant memories which are key factors contributing to the maintenance of several psychiatric disorders. Anxiety disorders like post-traumatic stress disorder (PTSD) are hallmarked by persistent, involuntary memory intrusions which are related to the traumatic event(s) and which occur along with distressing psychophysiological symptoms of arousal. Understanding how such disturbing emotional memories might be altered is currently a major research focus. Persistent, maladaptive memory is also central to drug addiction. In addiction, adaptive associative memory processes which normally direct attention and action to natural rewards like food or sex become usurped by drugs. The strong associations formed between drugs and environmental stimuli (cues) associated with drug use are known to promote habitual drug taking and craving and are a primary trigger of relapse.

Behavioural therapies traditionally aiming to weaken the influence of environmental cues on fear or drug cues on drug use have been based on extinction, whereby cue exposure occurs repeatedly in the absence of the conditioned response to the feared stimulus (e.g. electric shock). Similarly, the association between drug cues and drug use is extinguished by cue exposure in the absence of the unconditioned stimulus of the drug in animal models of addiction. In clinical practice, however, cue-exposure therapies for substance dependence have had limited efficacy, although attempts have been made to facilitate extinction learning notably by administering one or two doses of D-cycloserine (DCS) alongside extinction. Extinction learning inhibits, but does not overwrite, maladaptive memory traces. As such, learned associations between cues and drug use can persist and trigger relapse to drug use months or even years after abstention.

These limits of extinction-based therapies strengthen the appeal of intervening to reduce cue-conditioned response associations at the level of reconsolidation. By presenting an organism with cues that stimulate retrieval of the fear-related or drug-related memory and administering a beta-blocker (e.g. propranolol) or an NMDA antagonist (e.g. memantine, acamprosate, dexanabinol), the memory should subsequently be weakened (Das et al. 2013; Parsons and Ressler 2013).

Despite broad support for the existence of reconsolidation in several species across a range of learning paradigms and using various memory-blocking manipulations, results have been inconsistent. Whether reconsolidation occurs depends on a range of parameters including how memories are reactivated, whether novelty is introduced during reactivation and the age and strength of the memory (cf. Mackenzie and Eichenbaum 2011). These parameters form what is known as the boundary phenomena of reconsolidation. Although evidence for reconsolidation in non-human animals has accumulated rapidly in the last 10 years, as yet the evidence for human reconsolidation is relatively scant. This is partly because of the fact that many of the treatments administered to animals (e.g. protein synthesis inhibitors) are toxic to humans.

It is appropriate therefore that the next article in this Special Edition is a methodological review which covers both animal and human research. Auber et al. review the literature on post-retrieval extinction whereby extinction occurs after retrieval and during the reconsolidation window. Studies of both fear-related memories and drug-related memories lead to the conclusion that even slight alterations in methods can lead to significant differences in study findings. Auber et al. stress the importance of studying the boundary conditions under which memories can be reconsolidated. Reichelt and Lee's article also relates to methodological issues in addressing whether weakening the value of a specific conditioned stimulus via a Pavlovian overexpectation task is able to evoke memory destabilisation and thus reconsolidation of the memory.

The next seven articles concern reconsolidation, extinction and drug addiction. Torregrossa and Taylor's constructive review begins with the role of drug cues as powerful motivators of drug use behaviours which grab the user's attention and, even following abstention, can overshadow other reward cues and trigger relapse. They argue that reducing the strength of these associations can best be obtained via a combination of (1) enhancing extinction learning and (2) inhibiting drug-cue/drug-memory reconsolidation and that this combination holds significant promise for the treatment of addictive disorders.

Ledesma and Aragon use a conditioned place preference (CPP) paradigm in mice to determine how the $\mathrm{H}_{2} \mathrm{O}_{2}$ scavenger, alpha lipoic acid, affects the acquisition and reconditioning of ethanol-induced CPP. Three articles then focus on cocaine seeking in rats. Mahler et al. investigate how orexin and glutamate interact within the ventral tegmental area to promote the reinstatement of cocaine seeking in a rat self-administration paradigm. The influence of abstinence and intervals between extinction trials on cocaine-CPP in adolescent rats is the focus of Poltyrev and Yaka's study. Alaghband and Marshall again use a CPP paradigm in rats to directly compare the effects of two NMDA receptor antagonists (memantine and MK-801) on consolidation and reconsolidation of cocaine-cue memory. Their findings suggest that NMDA receptors are involved in both consolidation and reconsolidation, adding to the literature on the shared neural substrates of these two processes.

Saladin et al.'s article then reports a study of cocainedependent humans. They examined the effects of postretrieval propranalol on reconsolidation of memory for 
cocaine craving and cocaine-cue reactivity. Their cocaine or crack-dependent participants, currently abstinent, were first exposed to coke or crack videos and in vivo cocaine cues (e.g. pipes, white powder, paper money). Having reactivated memory, they were given either $40 \mathrm{mg}$ propranalol or placebo. At test $24 \mathrm{~h}$ later, those previously given the active drug showed lower cravings and less reactivity to cocaine cues than those given placebo, but 7 days later, there was no group difference. As Saladin et al. point out, their one-off intervention was minimal compared with the years of cocaine-cue consolidation that characterised their participants. It might be that multiple reconsolidation interventions would be needed to prolong the clinical effect seen a day after treatment.

Price et al. also report a study of cocaine-dependent participants. They expand the work on how DCS, a partial NMDA antagonist, might enhance the extinction of conditioned responses by facilitating extinction of drug cue reactivity. They add to the growing literature showing that DCS has no effect compared with placebo on reduction of craving; indeed, there was some elevation of craving following active treatment.

The final section of this Special Issue focuses on stress, consolidation and reconsolidation. Akirav and Maroun review the literature on how stress modulates reconsolidation. The ability of stress exposure to disrupt or facilitate the reconsolidation of emotional and drug-related memories has important implications for the treatment of PTSD and addictive disorders. They review evidence of brain areas involved and question the conditions under which stress would facilitate as opposed to disrupt reconsolidation. Jackson et al. aimed to determine whether a kappa opioid receptor antagonist would block stress-induced reinstatement of nicotine preference in a rat CPP model. Their findings of reduced reinstatement suggest the kappa opioid system as an appropriate therapeutic target for tobacco smokers.

Dongaonkar et al. explore how psychosocial stress affects episodic memory updating. Stress-induced increases in glucocorticoid and catecholamine release can suppress the function of memory-related brain regions and lead to impairments in retrieval. Studies of effects of stress on consolidation in humans depend on the paradigms, materials (e.g. emotional versus neutral; drug-related cues versus other reward-related cues) and participant characteristics. Their study used a verbal interference paradigm to explore the effects of stress (public speaking and mental arithmetic) on two phases of reconsolidation: reactivation and re-stabilisation. Stress prior to reactivation did not appear to impair memory updating, but stress after reactivation and the learning of the interference (second) list did. They conclude that the effects of stress on memory updating depend on when stress occurs: the closer it is to the onset of recon, the greater the impact.

The final article by Das et al. explores the effects of the cannabinoid, cannabidiol (CBD), in human contextual fear conditioning on the basis that $\mathrm{CBD}$ has been shown to enhance extinction in a rodent model of conditioned fear (Bitencourt et al. 2008). Given post-extinction, CBD potentiated the consolidation of extinction learning. Thus, this study suggests the endocannabinoid system should be a novel target in understanding how to enhance extinction. Further, CBD may be a useful adjunct to extinction-based psychological therapies in anxiety disorders.

Overall, the articles in the Special Issue pinpoint a wide range of methodological issues confronting studies of reconsolidation and the need to define boundary conditions under which reconsolidation reliably occurs. This will aid progress in translation efforts to use memory-based techniques in the clinic with patients with anxiety disorders and/or addictive disorders. The therapeutic potential of blocking reconsolidation and enhancing extinction provides a potential mechanism for ameliorating maladaptive associative memories in addiction which may importantly prevent relapse.

\section{References}

Bitencourt RM, Pamplona FA, Takahashi RN (2008) Facilitation of contextual fear memory extinction and anti-anxiogenic effects of AM404 and cannabidiol in conditioned rats. Eur Neuropsychopharmacol 18:849-859

Das RK, Freeman TP, Kamboj SK (2013) The effects of N-methyl Daspartate and B-adrenergic receptor antagonists on the reconsolidation of reward memory: a meta-analysis. Neurosci Biobehav Rev 37:240 255

Dudai Y (2006) Reconsolidation: the advantage of being refocused. Curr Opin Neurobiol 16(2):174-178

Hardt OH, Einarsson EO, Nader K (2010) A bridge over troubled water: reconsolidation as a link between cognitive and neuroscientific memory research traditions. Annu Rev Psychol 61:141-167

Mackenzie S, Eichenbaum H (2011) Consolidation and reconsolidation: two lives of memories? Neuron 71:224-232

McGaugh JL (1966) Time dependent processes in memory storage. Science 153:1351-1358

McGaugh JL (2000) Memory - a century of consolidation. Science 287:248-251

Milton AL, Everitt BJ (2012) The persistence of maladaptive memory: addiction, drug memories and anti-relapse treatments. Neurosci Biobehav Rev 36(4):1119-1139

Muller GE, Pilzecker A (1900) Experimentale Beitrage zur Lehre vom Gedachtis. Z Psychol Erganzungsband (suppl. no. 1):1-300

Nader K, Schafe GE, Le Doux JE (2000) Fear memories require protein synthesis in the amygdala for reconsolidation after retrieval. Nature 406:722-726

Osan R, Chen G, Feng R, Tsien JZ (2011) Differential consolidation and pattern reverberations within episodic cell assemblies in the mouse hippocampus. PLoS One 6(2):e16507. doi:10.1371/ journal.pone. 0016507

Parsons RG, Ressler KJ (2013) Implications of memory modulation for post-traumatic stress and fear disorders. Nat Neurosci 16(2):146-153

Ribot T (1881) Les Maladies de la Memoire. Appletron-CenturyCrofts, New York

Sevenster D, Beckers T, Kindt M (2013) Prediction error governs pharmacologically induced amnesia for learned fear. Science 339:830-833

Squire LR, Alvarez P (1995) Retrograde amnesia and memory consolidation: a neurobiological perspective. Curr Opin Neurobiol 5:169-177 